\title{
Analysis of Facial Features according to Sasang Types between Native Japanese and Native Korean Populations
}

\author{
Lin Ang, ${ }^{1,2}$ Jong Yeol Kim $\mathbb{D}^{1,2}$ and Jeongyun Lee $\mathbb{D}^{3}$ \\ ${ }^{1}$ Korea Institute of Oriental Medicine (KIOM), 1672 Yuseong-daero, Yuseong-gu, 34054 Daejeon, Republic of Korea \\ ${ }^{2}$ Korean Medicine Life Science, University of Science and Technology, 217 Gajeong-ro, Yuseong-gu, 34113 Daejeon, Republic of Korea \\ ${ }^{3}$ Division of Clinical Medicine, School of Korean Medicine, Pusan National University, 49 Busandaehak-ro, Mulgeum-eup, \\ Yangsan-si, 50612 Gyeongsangnam-do, Republic of Korea
}

Correspondence should be addressed to Jeongyun Lee; prajnamoon@gmail.com

Received 23 April 2018; Accepted 18 July 2018; Published 1 August 2018

Academic Editor: Sang-Hoon Shin

Copyright (C) 2018 Lin Ang et al. This is an open access article distributed under the Creative Commons Attribution License, which permits unrestricted use, distribution, and reproduction in any medium, provided the original work is properly cited.

Background. Facial diagnosis is a common practice and essential diagnostic method used in the Sasang Constitution Medicine (SCM). SCM is a kind of personalized medicine in Traditional Korean Medicine which categorizes people into four types, namely, Tae-Yang (TY) type, Tae-Eum type (TE), So-Yang (SY) type, and So-Eum (SE) type. This study was conducted to compare and analyze the differences in the facial feature across Sasang types among native Japanese and native Koreans. Methods. A total of 843 subjects were recruited for this study, 127 native Japanese and 716 native Koreans, respectively. Facial feature points and the measurements of facial features were assigned and calculated automatically using a facial analysis program. Data of each Sasang type for both genders were also extracted and analyzed. Analysis of covariance was then used to examine the differences in facial feature variables among native Japanese and native Koreans and Sasang types. Results. Significant differences were seen in the facial feature variables related to lower face area and eye shape. In males, TE types had wider mid-face and lower face as compared to other constitutions. Male TE types were also seen to have narrower eyes whereas male SY types had rounder eyes. In females, TE types had wider lower face width and area compared to SY types and SE types. Female SY types also had rounder eyes. Conclusions. This study presented distinctive feature in the lower face area and eye shape among the Sasang types in both native Japanese and native Koreans. This proposed that facial feature variables can also be used as an objective tool in distinguishing the Sasang types in native Japanese. Further studies are needed in the future to generalize these results.

\section{Background}

Sasang Constitutional Medicine (SCM) is a kind of typological personalized medicine in Traditional Korean Medicine which categorized people into four types, Tae-Yang (TY) type, Tae-Eum (TE) type, So-Yang (SY) type, and So-Eum (SE) type. This theory was established and written in the classic Longevity and Life Preservation in Eastern Medicine by Lee Jema around the year 1894 [1]. According to this classic, the determination of Sasang type comprised several diagnosis elements such as facial appearance, type of body shape, biopsychological traits, pathophysiological symptom diagnosis, and type-specific clinical response [2-4].

In the midst of those diagnosis elements, facial appearance can be regarded as one of the most important aspects
[5]. Although SCM classics had descriptions of the facial characteristics of the four Sasang types, those statements are relatively subjective and less quantitative $[1,6-13]$. Therefore, many efforts had been made to standardize and objectify the diagnosis of Sasang types using facial features [14-22].

In addition, SCM specialists had strongly accounted for facial features as one of the most reliable elements in distinguishing Sasang types [23]. Many studies had been conducted and facial features were shown to be highly employed in the determination of Sasang types $[6,16,24-$ 26]. Another study had also mentioned that face shape was found to be more utilized among all the facial elements. Recently, a standardization approach on the facial diagnosis of Sasang types according to quantitative analysis of facial features was established and the representative facial images 
of each Sasang type among native Koreans were made public in the year $2012[5,27]$.

The purpose of this study was to expand the usage of facial features in the determination of Sasang type towards other ethnicities. We investigated and compared the facial features among native Japanese and native Koreans across Sasang types by collecting their facial photographs and analyzing them quantitatively.

\section{Methods}

\subsection{Subjects}

2.1.1. Native Japanese (Sample A). This study was conducted at Tohoku University, Sendai, Japan, from 2010 to 2011 after receiving approval from the Institutional Review Board of Tohoku University where a total of 127 native Japanese were recruited. All the subjects who were eligible met the following inclusion criteria: (1) age ranging from 18 to 40 years and (2) being healthy and not suffering from chronic diseases. Subjects who have undergone plastic surgery or facial reconstruction surgery due to trauma were excluded. Male subjects with excessive facial hair were also excluded. A written consent form was signed by all the subjects before participating in this study.

2.1.2. Native Korean Subjects (Sample B). Data collected by Korea Constitutional Multicenter Bank (KCMB) between 2007 and 2010 were used in this study. Data of 716 native Koreans whom their age ranged from 18 to 40 years were extracted and analyzed. This procedure was done with the approval of the Korean Institute of Oriental Medicine Institutional Review Board (I-0910/02-001).

2.2. Classification of Sasang Types. The determination of Sasang types for both native Japanese and native Korean was performed by trained experts using Sasang Constitutional Analysis Tool (SCAT) under standard operating procedures, where the SCAT system analyzed the combination of information on facial images, body shape, voice, and questionnaires [20, 22]. In terms of questionnaires, the Korean language questionnaires developed for the use of SCAT were translated into Japanese language and the reliability assessment of the questionnaire was performed [28].

In order to further confirm the Sasang constitutions of the subjects, the Sasang constitutions of native Japanese subjects were confirmed by a certified Sasang medicine specialist (JY Kim) who has more than 8 years of clinical experience accompanied by a professional translator whereas Sasang constitutions of native Korean subjects were then further determined based on the response of subjects towards Sasang type-specific herbal medicine. Sasang constitution of the native Korean subjects was confirmed by a certified Sasang medicine specialist when they showed improvements in ordinary symptoms and did not suffer from adverse effects after taking the prescription for 50 or more days.

2.3. Facial Photography. Frontal full face and profile pictures are essential and should be taken with a neutral facial expression for all subjects. Pictures were taken at a fixed subject-camera distance of $1.6 \mathrm{~m}$ using a Nikon D700/D5100 digital camera with $85 \mathrm{~mm}$ lens under bilateral illumination. The camera was maintained at the same height as each subject. Images were taken at a resolution of $3184 \times 2120$ pixels in JPEG format using 24-bit RGB encoding. As for the subjects, ears and hairline of each subjects have to be revealed using a hair tie or hair band during the photography shoot. A ruler used for converting pixels into millimeters was placed approximately $1 \mathrm{~cm}$ below the chin. The first shot is the full face frontal view. Subjects were instructed to look at the lens of the camera with their heads positioned in a way that the central point of the two pupils and the upper auricular points were horizontal. The next shot is the profile view where the subject's face is turned approximately 90 degrees from the front. Only one side of their face and not the eye on the far side should be seen. The central point of the pupil from the side and point of upper auricular should also be on the same horizontal line.

2.4. Measurement of Facial Feature. The facial feature points were automatically allocated by uploading the facial images into the Sasang Constitutional Analysis Tool (SCAT) as shown in Figure 1. Facial feature variables were also automatically calculated using length, length to length ratio, angle, and area between facial feature points (Table 1).

2.5. Statistical Analysis. Data analysis of facial features was conducted independently for each sample according to gender. The differences in the general characteristics (age, height, weight, and BMI) of both samples were tested using Student's $t$-test. The differences in general characteristics (age, height, weight, and BMI) of Sasang types for native Koreans were tested using one-way ANOVA with Bonferroni or Dunnett's T3 as post hoc analysis, depending on the result of Levene's test. For native Japanese, general characteristics (age, height, weight, and BMI) of Sasang types were tested with Kruskal-Wallis test with Mann-Whitney as post hoc analysis. Statistical results were presented as mean (standard deviation).

The facial features of the samples were analyzed according to the Sasang types using one-way ANCOVA, with age as covariates and sample and Sasang types as factors. Post hoc analysis was performed with a Bonferroni adjustment. Statistical results were presented as adjusted mean (standard error).

Statistical analyses were performed using IBM SPSS Statistics 23.0 for Windows (IBM, Armonk, New York) at the significant level of 0.05 as the $p$ value.

\section{Results}

3.1. General Characteristics of the Subjects. The general characteristics of the subjects by samples were shown in Table 2. The mean age of native Japanese (Sample A) was $23.8 \pm 4.4$ years (ranging from 20 to 40 years old) for males and $24.2 \pm$ 5.4 years (ranging from 19 to 40 years old) for females. The mean age of native Koreans (Sample B) was 31.2 \pm 6.1 years (ranging from 18 to 40 years old) for males and $31.4 \pm 5.9$ 
TABLE 1: Description of facial feature variables.

Variables

FD $n 1 \_n 2$ [or PD $\left.n, \_n 2\right]$

$\mathrm{FDH} \_n, \_n 2$ [or PDH_n1_n2]

FDV_n1_n2 [or PDV_n1_n2]

FDL_n1_n2_n3 [or PDL_n1_n2_n3]

FHD_n1_n2_n3_n4 [or PHD_n1_n2_n3_n4]

FDH_n1_n2_n3_n4 [or PDH_n1_n2_n3_n4]

FDD_n1_n2_n3_n4 [or PDD_n1_n2_n3_n4]

FVD_n1_n2_n3_n4 [or PVD_n1_n2_n3_n4]

FVV_n1_n2_n3_n4 [or PVV_n1_n2_n3_n4]

FVH_n1_n2_n3_n4 [or PVH_n1_n2_n3_n4]

FA_n1_n2 [or PA_n1_n2]

FAs_n1_n2 [or PA_n1_n2]

FAi_n1_n2 [or PAi_n1_n2]

FAis_n1_n2 [or PAis_n1_n2]

FA_n1_n2_n3 [or PA_n1_n2_n3]

FArea02

FArea03
Description

The length between two points in the frontal (side) picture The horizontal length between two points in the frontal (side) picture

The vertical length between two points in the frontal (side) picture

The length between the point $n 1$ and segments $n 2, n 3$

FDH_n1_n2 / FD_n3_n4 [or PDH_n1_n2 / PD_n3_n4]

FD_n1_n2 / FDH_n3_n4 [or PD_n1_n2 / PDH_n3_n4]

FD_n1_n2 / FD_n3_n4 [or PD_n1_n2 / PD_n3_n4]

FDV_n1_n2 / FD_n3_n4 [or PDV_n1_n2 / PD_n3_n4]

FDV_n1_n2 / FDV_n3_n4 [or PDV_n1_n2 / PDV_n3_n4]

FDV_n1_n2 / FDH_n3_n4 [or PDV_n1_n2 / PDH_n3_n4]

The angle that the straight-line vector $(n 1, n 2)$ makes with the horizontal line in the frontal (side) image

180 - The angle that the straight-line vector $(\mathrm{n} 1, \mathrm{n} 2)$ makes with the horizontal line in the frontal (side) image

The angle that the straight-line vector $(\mathrm{n} 1, \mathrm{n} 2)$ makes with the horizontal line in the frontal (side) image $*(-1)$

180 - The angle that the straight-line vector $(\mathrm{n} 1, \mathrm{n} 2)$ makes with the horizontal line in the frontal (side) image

The angle formed by the three points $\mathrm{n} 1, \mathrm{n} 2$, and $\mathrm{n} 3$ in the frontal (side) image

The area of the face defined using points 53, 94, 194, and 153

The area of the face defined using points 94, 43, 143, and 194

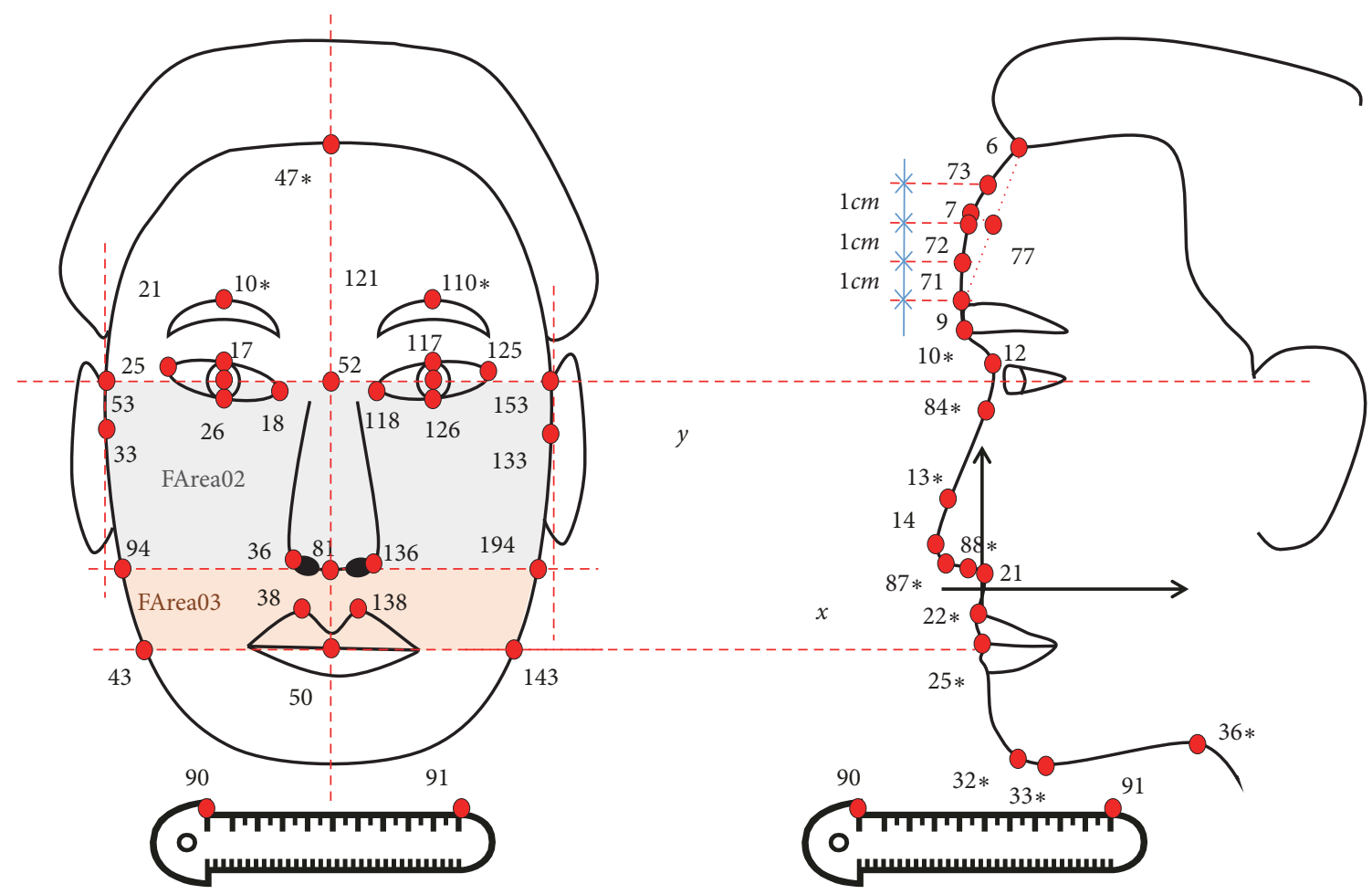

FIGURE 1: Automatically allocated facial feature points using facial analysis program [29]. 
TABLE 2: General characteristics of the subjects by samples.

\begin{tabular}{|c|c|c|c|c|c|c|}
\hline & \multicolumn{3}{|c|}{ Males } & \multicolumn{3}{|c|}{ Females } \\
\hline & $\begin{array}{c}\text { Sample A } \\
(\mathrm{n}=61)\end{array}$ & $\begin{array}{l}\text { Sample B } \\
(\mathrm{n}=252)\end{array}$ & $P$ value & $\begin{array}{c}\text { Sample A } \\
(\mathrm{n}=66)\end{array}$ & $\begin{array}{l}\text { Sample B } \\
(\mathrm{n}=464)\end{array}$ & $P$ value \\
\hline Age (yrs) & $23.83(4.39)$ & $31.18(6.08)$ & $<0.001$ & $24.19(5.36)$ & $31.43(5.93)$ & $<0.001$ \\
\hline Height $(\mathrm{cm})$ & $170.80(6.06)$ & $173.50(5.67)$ & 0.001 & $158.67(5.28)$ & $160.92(5.18)$ & 0.001 \\
\hline Weight (kg) & $64.93(9.13)$ & $72.54(11.23)$ & $<0.001$ & $52.27(6.37)$ & $55.81(8.89)$ & $<0.001$ \\
\hline BMI $\left(\mathrm{kg} / \mathrm{m}^{2}\right)$ & $22.20(2.48)$ & $24.36(3.14)$ & $<0.001$ & $20.75(2.22)$ & $21.55(3.24)$ & 0.012 \\
\hline
\end{tabular}

Sample A: native Japanese, Sample B: native Koreans, and BMI: Body Mass Index.

Data were presented as the mean (standard deviation). $P$ values were calculated using $t$-test.

TABLE 3: General characteristics of the subjects by Sasang types.

(a) Sample A

\begin{tabular}{|c|c|c|c|c|c|c|c|c|}
\hline \multirow[t]{2}{*}{ Sample A $(\mathrm{n}=127)$} & \multicolumn{4}{|c|}{ Male $(\mathrm{n}=61)$} & \multicolumn{4}{|c|}{ Female $(n=66)$} \\
\hline & $\mathrm{TE}(\mathrm{n}=17)$ & $\mathrm{SE}(\mathrm{n}=35)$ & SY $(n=9)$ & $P$ value & $\mathrm{TE}(\mathrm{n}=9)$ & $\mathrm{SE}(\mathrm{n}=19)$ & $\mathrm{SY}(\mathrm{n}=38)$ & $P$ value \\
\hline AGE (yrs) & $22.34(1.72)$ & $24.03(4.66)$ & $25.84(6.08)$ & 0.320 & $22.82(4.29)$ & $22.71(4.78)$ & $25.26(5.70)$ & 0.127 \\
\hline Height $(\mathrm{cm})$ & $174.07(5.47)^{\mathrm{a}}$ & $169.42(6.42)^{b}$ & $169.99(3.01)$ & 0.022 & $158.27(7.23)$ & $158.82(4.58)$ & $158.70(5.23)$ & 0.855 \\
\hline Weight (kg) & $72.30(9.27)^{\mathrm{a}}$ & $60.60(6.72)^{\mathrm{b}}$ & $67.82(7.34)^{\mathrm{a}}$ & $<0.001$ & $60.58(6.66)^{\mathrm{a}}$ & $48.32(4.56)^{c}$ & $52.28(5.18)^{\mathrm{b}}$ & $<0.001$ \\
\hline BMI $\left(\mathrm{kg} / \mathrm{m}^{2}\right)$ & $23.85(2.76)^{\mathrm{a}}$ & $21.08(1.72)^{b}$ & $23.46(2.30)^{\mathrm{a}}$ & $<0.001$ & $24.14(1.56)^{\mathrm{a}}$ & $19.15(1.55)^{\mathrm{c}}$ & $20.75(1.69)^{\mathrm{b}}$ & $<0.001$ \\
\hline
\end{tabular}

(b) Sample B

\begin{tabular}{|c|c|c|c|c|c|c|c|c|}
\hline \multirow[t]{2}{*}{ Sample B $(\mathrm{n}=716)$} & \multicolumn{4}{|c|}{ Male $(\mathrm{n}=252)$} & \multicolumn{4}{|c|}{ Female $(\mathrm{n}=464)$} \\
\hline & $\mathrm{TE}(\mathrm{n}=101)$ & $\mathrm{SE}(\mathrm{n}=79)$ & $S Y(n=72)$ & $P$ value & $\mathrm{TE}(\mathrm{n}=145)$ & $\mathrm{SE}(\mathrm{n}=156)$ & SY $(n=163)$ & $P$ value \\
\hline $\operatorname{AGE}(y)$ & $31.63(6.12)$ & $30.85(5.89)$ & $30.94(6.27)$ & 0.670 & $30.83(6.23)$ & $31.86(5.77)$ & $31.53(5.81)$ & 0.326 \\
\hline Height $(\mathrm{cm})$ & $174.26(5.72)$ & $172.70(5.92)$ & $173.28(5.30)$ & 0.212 & $161.41(4.98)$ & $161.03(5.07)$ & $160.41(5.44)$ & 0.239 \\
\hline Weight (kg) & $79.37(10.73)^{\mathrm{a}}$ & $66.52(9.76)^{b}$ & $69.66(8.22)^{b}$ & $<0.001$ & $63.00(9.42)^{\mathrm{a}}$ & $51.03(5.21)^{\mathrm{c}}$ & $54.19(7.20)^{\mathrm{b}}$ & $<0.001$ \\
\hline BMI $\left(\mathrm{kg} / \mathrm{m}^{2}\right)$ & $26.09(2.83)^{\mathrm{a}}$ & $22.26(2.70)^{b}$ & $23.18(2.36)^{b}$ & $<0.001$ & $24.20(3.59)^{\mathrm{a}}$ & $19.67(1.74)^{\mathrm{C}}$ & $21.05(2.43)^{\mathrm{b}}$ & $<0.001$ \\
\hline
\end{tabular}

Sample A: native Japanese, Sample B: native Koreans; TE: Tae-Eum, SE: So-Eum, SY: So-Yang.

Data were presented as the mean (standard deviation).

Sample A: $P$ values were calculated using Kruskal-Wallis test. Post hoc comparisons using Mann-Whitney.

Sample B: $P$ values were calculated using one-way ANOVA. Post hoc comparisons using Bonferroni or Dunnett T3.

$\mathrm{a}, \mathrm{b}, \mathrm{c}$ : Significant difference between the groups, in which the value descends by a and $\mathrm{b}$ followed by $\mathrm{c}$.

years (ranging from 18 to 40 years old) for females. In both males and females, age, height, body weight, and BMI were significantly higher in Sample B than in Sample A.

The general characteristics of the samples' Sasang types were shown in Table 3. There were no significant differences in age and height among the Sasang types for both genders in Sample B and females only in Sample A. For males in Sample A, there was no significant difference in age but there were significant differences in height. However, there were significant differences in weight and BMI among the Sasang types for males and females in both samples. Weight and BMI for both samples were the highest in TE types and lowest in SE types according to post hoc analysis.

\subsection{Differences in Facial Features among the Samples and} Sasang Types. After adjustment for age, there were several statistically significant differences in the facial features variables among samples and Sasang constitution in both genders.

3.2.1. Difference in Facial Features in Males. In males, lower face angle and area variables such as FA_18_17_43,
FA_118_117_143, and FArea03_aD showed significant differences between the samples and also among the Sasang constitutions. Lower face area was larger in Sample B and also in the TE types as compared to SE types and SY types. Midface area variable, FArea02_aD, was also seen significantly different where the mid-face of Sample B is larger than Sample A. The value of this variable was also highest in TE types compared to SE types and SY types (Table 4).

There was also a significant difference in the eye horizontal distance variable, FDH_25_125, and also in the variables related to eye slanting angle, FA_18_17_25 and FA_118_117_125. This showed that the outer eye horizontal distance and eye slanting angle were larger in Sample B and also in TE types. Moreover, inner eye slanting angle, FAis_18_17 and FAi_118_117, was larger in Sample A and also in SY types as compared to other Sasang types (Table 4).

Forehead related variable, PD_7_77, was also significantly different across samples and Sasang types. The forehead of Sample A and SY types was more protruding than Sample $\mathrm{B}$ and other Sasang types. Furthermore, there is also a significant difference in the nose related variable such as PDH_12_14 where Sample A had shorter nose than Sample B. 
TABLE 4: Male facial feature variables with differences among the samples and Sasang types.

\begin{tabular}{|c|c|c|c|c|c|c|c|c|}
\hline \multirow[b]{2}{*}{ Facial variables } & \multicolumn{3}{|c|}{ Samples } & \multicolumn{4}{|c|}{ Sasang types } & \multirow{2}{*}{$\begin{array}{c}\text { Sample } * \\
\text { Sasang types } \\
P \text { value }\end{array}$} \\
\hline & Sample A & Sample B & $P$ value & $\mathrm{TE}$ & SE & SY & $P$ value & \\
\hline FA_18_17_43 & 77.55 (1.16) & $83.12(0.50)$ & $<0.001$ & $82.74(0.98)^{\mathrm{a}}$ & $80.68(0.77)^{\mathrm{ab}}$ & $77.58(1.29)^{\mathrm{b}}$ & 0.006 & 0.160 \\
\hline FA_118_117_143 & $78.39(1.08)$ & $82.81(0.47)$ & $<0.001$ & $82.96(0.92)^{\mathrm{a}}$ & $80.48(0.72)^{\mathrm{ab}}$ & $78.37(1.21)^{\mathrm{b}}$ & 0.008 & 0.079 \\
\hline FArea02_aD & $6787.01(114.31)$ & $7266.28(49.14)$ & $<0.001$ & $7245.54(96.90)^{\mathrm{a}}$ & $7064.10(76.28)^{\mathrm{ab}}$ & $6770.29(127.62)^{\mathrm{b}}$ & 0.012 & 0.074 \\
\hline FArea03_aD & $3831.44(72.95)$ & $4089.65(31.36)$ & 0.002 & $4119.47(61.84)^{\mathrm{a}}$ & $3948.55(48.68)^{\mathrm{ab}}$ & $3813.62(81.45)^{\mathrm{b}}$ & 0.008 & 0.288 \\
\hline FDH_25_125 & $97.86(0.92)$ & $104.46(0.39)$ & $<0.001$ & $102.63(0.78)^{\mathrm{a}}$ & $101.65(0.61)^{\mathrm{ab}}$ & $99.20(1.02)^{\mathrm{b}}$ & 0.027 & 0.123 \\
\hline FA_18_17_25 & $128.72(1.21)$ & $133.30(0.52)$ & 0.001 & $133.14(1.02)^{\mathrm{a}}$ & $131.89(0.81)^{\mathrm{a}}$ & $127.99(1.35)^{\mathrm{b}}$ & 0.009 & 0.162 \\
\hline FA_118_117_125 & $127.94(1.16)$ & $132.30(0.50)$ & 0.001 & $132.27(0.98)^{\mathrm{a}}$ & $130.84(0.77)^{\mathrm{ab}}$ & $127.26(1.29)^{\mathrm{b}}$ & 0.008 & 0.132 \\
\hline FAis_18_17 & $32.03(0.80)$ & $29.47(0.34)$ & 0.005 & $28.89(0.68)^{\mathrm{b}}$ & $30.52(0.53)^{\mathrm{ab}}$ & $32.85(0.89)^{\mathrm{a}}$ & 0.002 & 0.265 \\
\hline FAi_118_117 & $31.71(0.82)$ & $29.48(0.35)$ & 0.015 & $29.01(0.69)^{b}$ & $30.42(0.55)^{\mathrm{ab}}$ & $32.35(0.91)^{\mathrm{a}}$ & 0.014 & 0.230 \\
\hline PD_7_77 & $3.15(0.28)$ & $2.36(0.12)$ & 0.011 & $2.60(0.24)^{\mathrm{ab}}$ & $2.39(0.18)^{\mathrm{b}}$ & $3.28(0.31)^{\mathrm{a}}$ & 0.048 & 0.224 \\
\hline PDH_12_14 & $19.48(0.40)$ & $20.66(0.17)$ & 0.009 & $20.58(0.34)^{\mathrm{a}}$ & $20.53(0.27)^{\mathrm{a}}$ & $19.11(0.45)^{\mathrm{b}}$ & 0.015 & 0.099 \\
\hline
\end{tabular}

Sample A: native Japanese, Sample B: native Koreans; TE: Tae-Eum, SE: So-Eum, SY: So-Yang.

Data were presented as adjusted mean (standard error)

$P$ values were calculated using one-way ANCOVA. Post hoc comparisons using Bonferroni.

$\mathrm{a}, \mathrm{b}, \mathrm{c}$ : Significant difference between the groups, in which the value descends by a and b followed by $\mathrm{c}$.

ab: no significant difference among the groups.

TABLE 5: Female facial feature variables with differences among the samples and Sasang types.

\begin{tabular}{|c|c|c|c|c|c|c|c|c|}
\hline \multirow[b]{2}{*}{ Facial variables } & \multirow[b]{2}{*}{ Sample A } & \multicolumn{2}{|l|}{ Samples } & \multicolumn{4}{|c|}{ Sasang types } & \multirow{2}{*}{$\begin{array}{c}\text { Sample } * \\
\text { Sasang types } \\
P \text { value }\end{array}$} \\
\hline & & Sample B & $P$ value & $\mathrm{TE}$ & SE & SY & $P$ value & \\
\hline FDV_52_50 & $75.28(0.65)$ & $71.83(0.20)$ & $<0.001$ & $73.82(0.74)^{\mathrm{ab}}$ & $74.36(0.53)^{a}$ & $72.49(0.39)^{\mathrm{b}}$ & 0.011 & 0.105 \\
\hline FDV_81_50 & $28.45(0.38)$ & $26.07(0.12)$ & $<0.001$ & $27.76(0.43)$ & $27.42(0.30)$ & $26.62(0.22)$ & 0.018 & 0.103 \\
\hline FA_18_17_43 & $77.98(0.96)$ & $75.52(0.30)$ & 0.016 & $78.53(1.08)^{\mathrm{a}}$ & $75.10(0.77)^{\mathrm{b}}$ & $76.63(0.57)^{\mathrm{ab}}$ & 0.029 & 0.816 \\
\hline FArea03_aD & $3788.77(64.90)$ & $3525.75(20.10)$ & $<0.001$ & $3794.18(73.24)^{\mathrm{a}}$ & $3631.84(52.16)^{\mathrm{ab}}$ & $3545.76(38.50)^{\mathrm{b}}$ & 0.009 & 0.073 \\
\hline FD_17_26 & $8.89(0.18)$ & $10.02(0.06)$ & $<0.001$ & $9.03(0.21)^{\mathrm{b}}$ & $9.80(0.15)^{\mathrm{a}}$ & $9.53(0.11)^{\mathrm{ab}}$ & 0.010 & 0.090 \\
\hline FAis_18_17 & $31.57(0.69)$ & $35.51(0.22)$ & $<0.001$ & $32.41(0.78)^{\mathrm{b}}$ & $34.70(0.56)^{\mathrm{a}}$ & $33.52(0.41)^{\mathrm{ab}}$ & 0.044 & 0.620 \\
\hline
\end{tabular}

Sample A: native Japanese, Sample B: native Koreans; TE: Tae-Eum, SE: So-Eum, SY: So-Yang.

Data were presented as adjusted mean (standard error)

$P$ values were calculated using one-way ANCOVA. Post hoc comparisons using Bonferroni.

$\mathrm{a}, \mathrm{b}, \mathrm{c}$ : Significant difference between the groups, in which the value descends by a and b followed by $\mathrm{c}$.

ab: no significant difference among the groups.

For this variable, the noses of SY types were shorter than SE types (Table 4 ).

3.2.2. Difference in Facial Features in Females. In females, the facial variable related to the distance between the root of nose and lips, FDV_52_50, and philtrum related variable, FDV_81_50, showed significant differences among the samples. The values of these variables were larger in Sample A than Sample B which suggested that Sample A had longer nose and philtrum (Table 5).

Lower face angle and area variables, FA_18_17_43 and FArea03_aD, also showed significant differences among the samples and Sasang constitutions. This indicates that lower face width and area was overall wider in Sample A and in the TE types compared to the SY and SE types. There was also significant difference seen in variable FD_17_26, which is the distance between upper and lower eyelid in vertical alignment. Furthermore, variable related to the angle of eye slanting, FAis_18_17, also showed significant differences where
Sample B and SE types had a wider angle than Sample A and other Sasang types (Table 5).

\section{Discussion}

As the diagnosis of facial features is one of the fundamental aspects of SCM, understanding the differences in facial features among Sasang types is essential. Currently, the standard approach to Sasang types facial diagnosis among native Koreans has already been established using quantitative analysis. However, the use of Sasang types facial diagnosis in other populations is still limited. This study was performed to extend the use of Sasang facial diagnosis in native Japanese.

In this study, we analyzed the differences in facial feature variables among the samples and Sasang types. In terms of demographic characteristics, we found that the age, height, weight, and BMI were significantly higher in Sample B than in Sample A for both genders (Table 2). The average height and weight of our samples correspond with the average height and 
weight of both nationalities in general, which could suggest that the results in our study could speak for native Japanese and native Koreans in general [30-34]. Besides, TE types also had the highest weight and BMI followed by SE types as the lowest in both samples (Table 3). This shows that the results of Sample A are consistent with the previous study of Sample $\mathrm{B}$ where TE type was reported to have highest BMI score and it was lowest in SE type $[4,17,35]$.

After adjustment for age, facial variables such as FA_18_17_43, FArea03_aD, and FAis_18_17 were the common variables significantly different in both males and females. Therefore, lower face variables and eye-related variables were considered the main indicators that distinguish the Sasang types in both genders among both samples. The value of lower face angle and area variables, FA_18_17_43 and FArea03_aD, was higher in male and lower in the female of Sample B indicating that lower face of Sample B was larger than Sample A in male and vice versa in the female. However, the values of these variables were higher in TE type of both males and females (Tables 4 and 5). These results are similar to those previous studies that analyzed facial feature according to Sasang types among native Koreans. It was mentioned that the width over length was larger in TE type with the width of jaw serving as standard and generally TE type had a wider jaw than other Sasang types [16, 17, 25].

On the other hand, the value of the variable related to the area of mid-face, FArea02aD, was also higher in the male of Sample B and TE types (Table 4). This indicates that male TE types have larger mid-face. Looking at the results of both midface and lower face area variables for males, we can suggest that males of TE types have wider face area compared to other Sasang types. These results could be considered consistent with the previous studies where the face of TE types was mentioned to be larger than other types [16].

Furthermore, the value of eye slanting angle variables, FA_18_17_25 and FA_118_117_125, was higher in the males of Sample B compared to the males of Sample A and vice versa for the inner eye slanting angle variables, FAis_18_17 and FAi_118_117. This indicates that Sample A males have rounder eyes whereas Sample B males have narrower eyes. However, in females, the value of inner eye slanting angle variable FAis_18_17 and the value of variable FD_17_26, which is the vertical distance of upper and lower eyelid, were higher in Sample B (Tables 4 and 5). This shows that Sample A females have narrower eyes and Sample B females have rounder eyes. Our findings showed that the eye shape of both genders in Sample A and Sample B is opposite to each other.

In terms of Sasang types, the value of eye slanting angle variables, FA_18_17_25 and FA_118_117_125, is the highest in TE types compared to other types in males. This shows that TE types of males have narrower eyes. In addition, males of SY types and females of SE types have the highest value in the inner eye slanting angle variable, FAis_18_17, indicating that SY types males and SE types females have rounder eyes (Tables 4 and 5). A previous study had stated that female TE types had narrower eyes and female SE type had rounder eyelids [25]. Another study also suggested that the eye of SE type is round in shape and the eye of SY type was relatively rounder than TE types [16]. Our results showed similar consistency with previous studies. Moreover, the value of forehead related variable, PD_7_77, is highest in SY types according to our post hoc analysis. This finding also matched the descriptions of previous studies which mentioned that the SY types had the most protruding forehead and bulging head $[23,25]$.

We also found that there are a few significant results when we look only at the differences among the Sasang types without regard to the differences in samples. In males, there were significant differences in the variable PDV_14_21 among the Sasang types. This showed that TE types have longer vertical distance between the apex of nose and subnasale. This results indicated that, regardless of sample types, the nose of TE types males was more turn-up compared to other types.

In addition, the consistency between our study and previous studies implies that the algorithm used in Sasang Constitutional Analysis Tool for native Koreans can also be utilized in the analysis of Sasang types of native Japanese provided that the standardized values compensating for the difference of sample size are assigned to the algorithm [22]. Hence, further studies are needed to validate our findings.

There are several limitations in this study. Firstly, the determination of the subjects' Sasang types is inconsistent between the samples. Sasang types of the subjects in Sample B were decided based on their response towards Sasang typespecific herbal medicine while Sasang types of the subjects in Sample A were determined by only one certified clinical specialist and they were not prescribed Sasang type-specific herbal medicine. Therefore, there might be a question on the generality of data. Secondly, subjects in Sample B were chosen from data bank based on the predefined inclusion criteria whereas subjects in Sample A were recruited from one university, resulting in unequal sample size which may affect the ability to generalize our findings. Although we did necessary statistical adjustment for age, conclusion involving these data should be drawn with caution.

Hence, future studies should include a larger sample size from various places of Japan and Sasang types of subjects should be determined by at least two or more certified clinical specialists with their interrater reliability tested. The improvement in the reliability of data collection will greatly improve the accuracy of our research. Such follow-up studies will greatly generalize our findings and may be able to yield new discoveries.

\section{Conclusion}

This study is the first study which attempts to analyze the facial features of native Japanese individuals according to Sasang types. Although there were differences in facial features among Sample A and Sample B, the facial features of both samples across Sasang types showed a similar tendency. If the distinctive variables are applied after compensating for the differences between samples, the Sasang Constitutional Analysis Tool may be valid and usable in distinguishing the Sasang types of native Japanese. 


\section{Data Availability}

The data used to support the findings of this study are available from the corresponding author upon request.

\section{Disclosure}

The authors would also like to mention that this study was presented in part as conference abstract in the 2018 International Congress on Integrative Medicine and Health, Baltimore, Maryland, USA, May 2018 (Abstract 4039).

\section{Conflicts of Interest}

There are no conflicts of interest in this study.

\section{Acknowledgments}

This research was supported by the Bio \& Medical Technology Development Program of the National Research Foundation of Korea (NRF) funded by the Korean government, MSIP (NRF-2015M3A9B6027139).

\section{References}

[1] J. M. Lee and S. H. Choi, Longevity and Life Preservation in Oriental Medicine, Kyung Hee University Press, Seoul, 1966.

[2] H. Chae, I. K. Lyoo, S. J. Lee et al., "An alternative way to individualized medicine: psychological and physical traits of Sasang typology," The Journal of Alternative and Complementary Medicine, vol. 9, no. 4, pp. 519-528, 2003.

[3] J. Y. Kim and D. D. Pham, "Sasang constitutional medicine as a holistic tailored medicine," Evidence-Based Complementary and Alternative Medicine, vol. 6, supplement 1, pp. 11-19, 2009.

[4] S. J. Lee, S. H. Park, C. R. Cloninger, Y. H. Kim, M. Hwang, and H. Chae, "Biopsychological traits of Sasang typology based on Sasang personality questionnaire and body mass index," BMC Complementary and Alternative Medicine, vol. 14, article 315, 2014.

[5] S. Lee, B. Koh, E. Lee, J. Lee, and M. Hwang, "Systematic Review on Researches of Sasang Constitution Diagnosis Using Facial Feature," Journal of Sasang Constitutional Medicine, vol. 24, no. 4, pp. 17-27, 2012.

[6] E. H. Sohn, J. H. Yoo, J. W. Kim et al., "The Study of Sasangin's Face," Journal of Sasang Constitutional Medicine, vol. 17, no. 3, pp. 55-68, 2005.

[7] M. B. Lee, Keumgwe Bibang, HaeDong MunHwaSa, seuol, South Korea, 1999.

[8] D. R. Kim, Boje Yeonseol, Daeseong Publishing, seuol, South korea, 2002.

[9] Y. S. Kwon, Sasang Bangyak Happyeon, Haengrim Seowon, Seoul, South Korea, 1973.

[10] S. Y. Hong, Sasang Jinryo Bowon, Seowon Dang, Seoul, South Korea, 2002.

[11] I. S. Park, Dongui Sasang Yogyeol, Seoul, Sonamu, 2015.

[12] S. J. Huh, Sasang constitutional medicine, Yanbian University Press, Yanbian, 1998.

[13] J. Kim, Seong-ri Imsangron, Daeseong Publishing, Seoul, 1998.
[14] E. J. Lee, K. S. Kim, and E. H. Sohn, “The Study of Sasangin's Face by Sasangin Diagnosis Questionnaire(SDQ)," The Journal of Korean Medicine, vol. 27, no. 1, pp. 130-137, 2006.

[15] E. H. Kim, Y. J. Cho, and Y. H. Jung, "Anthropometric Facial Characteristics of Adult Tae-eumin of Northern and Southern Lineage in the Korean Peninsula," The Journal of Korean Medicine, vol. 30, no. 6, pp. 86-95, 2009.

[16] I. Koo, J. Y. Kim, M. G. Kim, and K. H. Kim, "Feature selection from a facial image for distinction of sasang constitution," Evidence-Based Complementary and Alternative Medicine, vol. 6, no. 1, pp. 65-71, 2009.

[17] D. D. Pham, J. H. Do, B. Ku, H. J. Lee, H. Kim, and J. Y. Kim, "Body Mass Index and Facial Cues in Sasang Typology for Young and Elderly Persons, Evidence-Based Complementary and Alternative Medicine," Evidence-Based Complementary and Alternative Medicine, vol. 2011, Article ID 749209, 9 pages, 2011.

[18] S. Lee and M. Hwang, "Studies on the Modeling of the Threedimensional Standard Face and Deriving of Facial Characteristics Depending on the Taeeumin and Soyangin," Journal of Sasang Constitutional Medicine, vol. 26, no. 4, pp. 350-364, 2014.

[19] S. K. Lee, E. J. Lee, B. H. Koh, I. B. Song, and J. H. Yun, "Morphological standardization research of head and face on the 50's and 60's in Korean according to Sasang Constitution," Journal of Sasang Constitutional Medicine, vol. 12, no. 2, pp. 123131, 2000.

[20] J. U. Kim, B. Ku, Y. M. Kim et al., "The Concept of Sasang Health Index and Constitution-Based Health Assessment: An Integrative Model with Computerized Four Diagnosis Methods," Evidence-Based Complementary and Alternative Medicine, vol. 2013, Article ID 879420, 13 pages, 2013.

[21] S.-W. Lee, E.-S. Jang, J. Lee, and J. Y. Kim, "Current Researches on the Methods of Diagnosing Sasang Constitution," Evidencebased Complementary and Alternative Medicine, vol. 6, supplement 1, pp. 43-49, 2009.

[22] J.-H. Do, E. Jang, B. Ku, J.-S. Jang, H. Kim, and J. Y. Kim, “Development of an integrated Sasang constitution diagnosis method using face, body shape, voice, and questionnaire information," BMC Complementary and Alternative Medicine, vol. 12, article 85, 2012.

[23] J. H. Lee, Y. H. Kim, and M. W. Hwang, "Survey Study about Sasangin's Characteristics of Face, Voice, Skin and Pulse Diagnosis," Journal of Sasang Constitutional Medicine, vol. 19, no. 3, pp. 123-143, 2007.

[24] J. W. Kim and S. H. Jeon, "A Study on the Characteristics of Facial Shape in Adult Women by Sasang Constitution Using Hyungsang Classification," Journal of Sasang Constitutional Medicine, vol. 29, no. 2, pp. 95-103, 2017.

[25] J. Do, B. Ku, J. Jang, H. Kim, and J. Y. Kim, "Analysis of Sasang constitutional types using facial features with compensation for photographic distance," Integrative Medicine Research, vol. 1, no. 1, pp. 26-35, 2012.

[26] Y. J. Park, J.-H. Do, H. Kim, and J. Y. Kim, "Differences in Complexion between Cold- and Heat-Prescription Groups in Sasang Medicine, Evidence-Based Complementary and Alternative Medicine," Evidence-Based Complementary and Alternative Medicine, vol. 2017, Article ID 9701978, 9 pages, 2017.

[27] Y. H. Yoo, "Am I a Tae-Eum type or a So-Yang type? Represent the standard facial features of each Sasang type," in Dong- $A$ Daily News, 2012.

[28] J. Yoo, E. Jang, Y. Kim, K. Park, and S. Lee, "A Study on the Reliability Assessment of Sasang Constitution Questionnaire 
Developed by KIOM for Japanese," Journal of Sasang Constitutional Medicine, vol. 24, no. 2, pp. 8-18, 2012.

[29] J. Nam, J. S. Jang, H. Kim, J. Y. Kim, and J. H. Do, "Modification of the Integrated Sasang Constitutional Diagnostic Mode," Evidence-Based Complementary and Alternative Medicine, vol. 2017, Article ID 9180159, 8 pages, 2017.

[30] Y. Lee, "Anthropometric Data Analysis for Body Shape Modeling in Korean," Korean Journal of Physical Anthropology, vol. 26, no. 2, p. 61, 2013.

[31] D. D. Pham, J. H. Lee, K. Y. Kim, J. Y. Song, J. E. Kim, and C. H. Leem, "Anthropometry-based estimation of body heat capacity in individuals aged 7-69 years: the Size Korea Survey 2010," Scientific Reports, vol. 8, no. 1, 2490 pages, 2018.

[32] "Society at a Glance 2009: OECD Social Indicators," Retrieved 2014.07.15.

[33] Official Statistics by Ministry of Education, Culture, Sports, Science and Technology,.

[34] "Official Statistics by Ministry of Education, Culture, Sports, Science and Technology. e-stat.go.jp," Retrieved 2012.02.11.

[35] K. H. Song, S. G. Yu, S. Cha, and J. Y. Kim, "Association of the Apolipoprotein A5 Gene -1131T>C Polymorphism with Serum Lipids in Korean Subjects: Impact of Sasang Constitution," Evidence-based Complementary and Alternative Medicine, vol. 2012, Article ID 598394, 8 pages, 2012. 


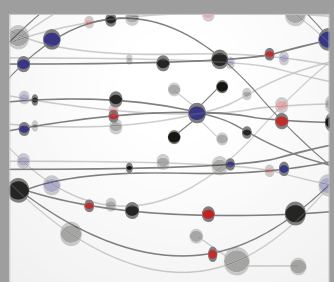

The Scientific World Journal
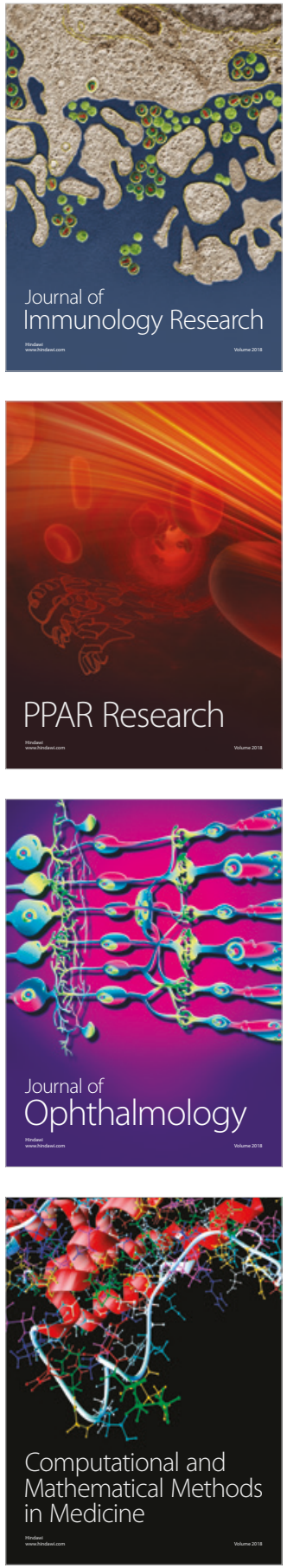

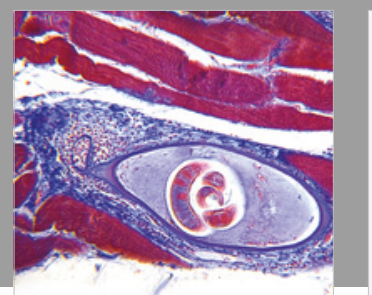

Gastroenterology Research and Practice

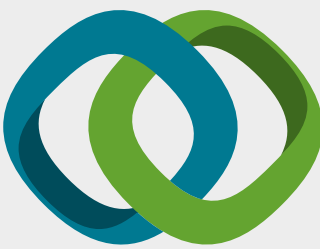

\section{Hindawi}

Submit your manuscripts at

www.hindawi.com
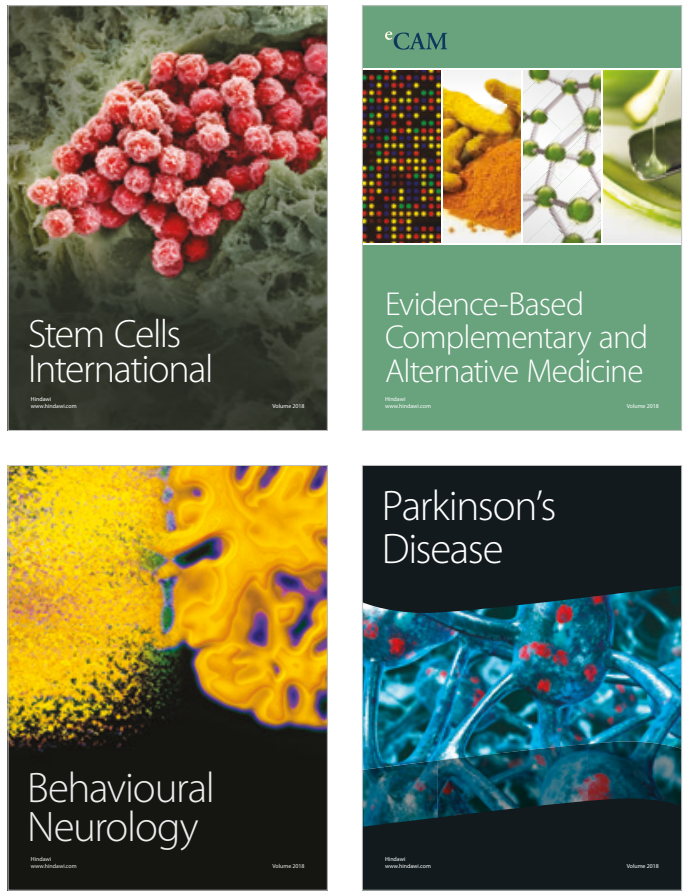

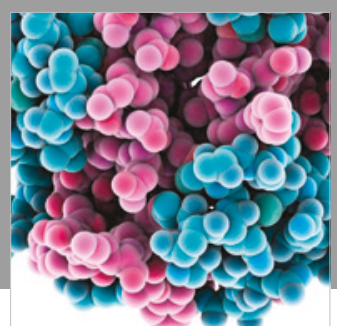

ournal of

Diabetes Research

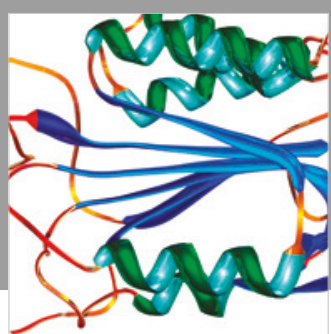

Disease Markers
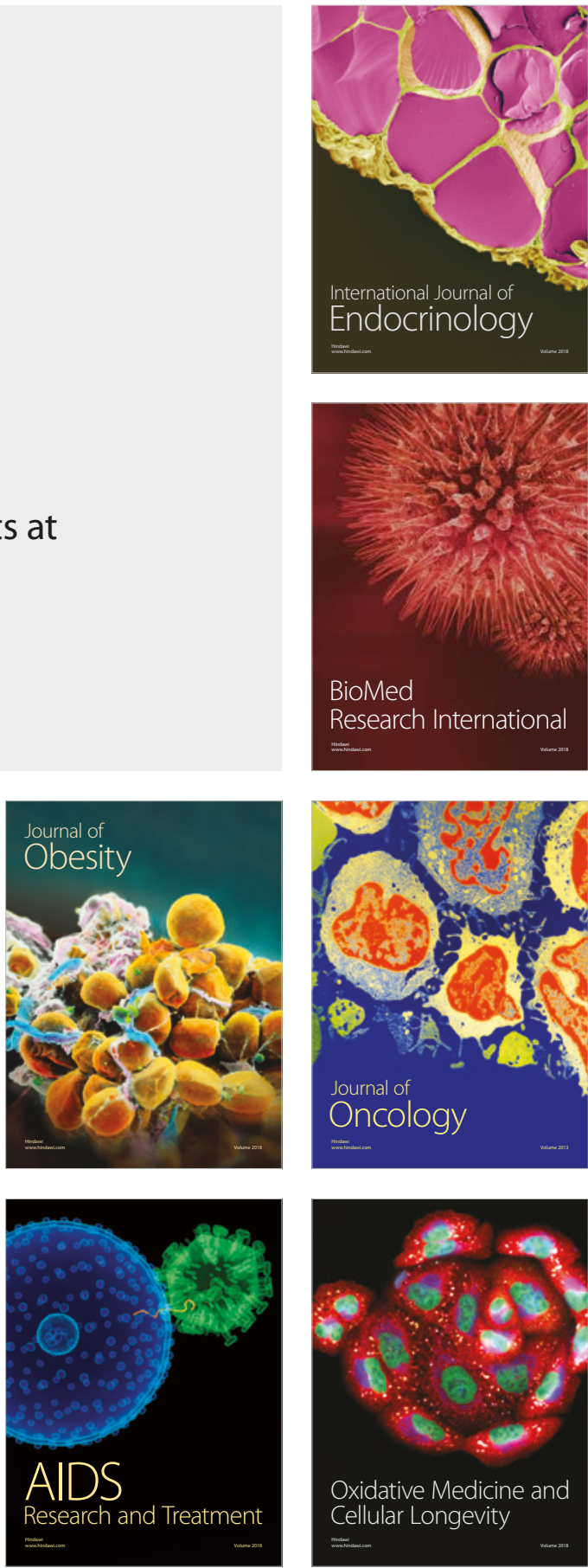